\title{
Correspondence
}

\section{The anesthetic record}

\section{To the Editor:}

The study by Devitt et al. ${ }^{1}$ addressing the accuracy and completeness of the anesthetic record adds to the literature supporting the shortcomings of written records. The anesthetic record is now scoured for research data, ${ }^{2}$ scrutinized for fiscal and cost containment information, ${ }^{3}$ monitored to ensure $\mathrm{CQI},{ }^{4}$ and exhibited to resolve medicolegal disputes. Can meaningful conclusions be made in these realms using a record generated with pen and paper? Accurate representation of hypotensive and hypoxic events in electronic records requires sampling intervals of $36 \mathrm{sec}$ and 13 sec respectively. ${ }^{5}$

Devitt's record resembles that of our centre. The grid portion of the chart allows the user to create the time axis. The tendency is to define one small box as a five minute interval thus comforming to the CAS guidelines for heart rate and blood pressure recording. The accurate graphical documentation of a simulated (or real) critical incident of five minute duration is very difficult. Boldly placed blood pressure and heart rate readings between the lines may compromise legibility. What readings should be recorded - minimum, maximum or "smoothed" values? Devitt did not display the chart's note area. Often these spaces are too small to allow detailed notes of an event. Until the widespread introduction of AIMS, manual charting will remain the norm. We would make the following suggestions:

1 Despite the study results showing no improvement in charting accuracy or completeness, we would still encourage the use of the trend function when charting retrospectively.

2 Expand the time scale of the graphical written record during critical incidents. Notes are necessary if events cannot be accurately described graphically.

Richard A. Cherry MD FRCPC

Steven L. Dain MD FRCPC

London, Ontario

\section{References}

1 Devitt JH, Rapanos T, Kurrek M, Coben MM, Shaw $M$. The anesthetic record: accuracy and completeness Can J Anesth 1999; 46: 122-8.
2 Gibby GL. Anesthesia information-management systems: their role in risk-versus cost assessment and outcomes research. J Cardiothorac Vasc Anesth 1997; 11(Suppl2): 2-5.

3 Lubarsky DA, Sanderson IC, Gilbert WC, et.al. Using an anesthesia information management system as a cost containment tool. Description and validation. Anesthesiology 1997; 86: 1161-9.

4 Edsall DW, Jones BR, Smith NT. The anesthesia database, the automated record and the quality assurance process. Int Anesthesiol Clin 1992; 30: 71-92.

5 Derrick JL, Bassin DJ. Sampling intervals to record severe hypotensive and hypoxic episodes in anesthetised patients J Clin Monit Comput 1998; 14: 347-51.

\section{REPLY:}

The letter by Cherry and Dain raises several important points. Our study supports a growing body of literature that suggests hand charting by anesthesiologists is inaccurate. ${ }^{1-4}$ As a result, the data recorded in the chart may be flawed and any use of this retrospective information could lead to erroneous conclusions.

Byrne and coworkers bave studied the accuracy of band charting when the time scale was expanded from 5 to 2.5 min blocks. Despite the change in time scale, more than $20 \%$ of the values charted were in error by more than $25 \%$ of the real value. ${ }^{2}$ Our anesthetic chart is a four-page folding document. The chart design presented in Figure 1 represents a portion of the inside left band page. There is a 4 $\times 9 \mathrm{~cm}$ comments box at the bottom of this page. The facing page (inside right hand page) contains an additional four-hour grid for charting and an additional $8 \times 18$ $\mathrm{cm}$ comments box at the bottom of the page. In our study when we collected the data for the charted value, we included not only what was recorded in the grid portion of the chart but also any values written in the comments box that corresponded to the critical incidents.

We agree with the suggestions of Cherry and Dain. However, despite these suggestions, caution should be exercised when using retrospective hand charted anesthesia data for research, quality improvement or medicolegal purposes as the data contained in charts is likely to be inaccurate and any conclusions from the above processes may be incorrect.

J. Hugh Devitt MD MSC FRCPC

Matt M. Kurrek MD

Toronto, Ontario 


\section{References}

1 Devitt JH, Rapanos $T$, Kurrek $M$, Coben $M M$, Shaw $M$. The anesthetic record: accuracy and completeness. Can J Anesth 1999; 46: 122-8.

2 Byrne AJ, Sellen AJ, Jones JG. Errors on anaesthetic record charts as a measure of anaesthetic performance during simulared critical incidents. Br J Anaesth 1998; 80: 58-62.

3 Edsall $D W$. Computerization of anesthesia information management-users' perspective. J Clin Monit 1991; 7 : 351-8.

4 Cook RI, McDonald JS, Nunziata E. Differences between handwritten and automatic blood pressure records. Anesthesiology 1989; 71: 385-90.

\section{PONV in Outpatients: when should it be assessed?}

To the Editor:

Despite major advances in anesthesia, postoperative nausea and vomiting (PONV) still remains a common distressing problem facing patients. In ambulatory care setting, it may lead to delayed discharge and unanticipated overnight admission, both with financial implications to both the patient and the health provider. Referring to the recent article by Dr Chung in the April edition of this journal, I would like to comment on the low incidence of PONV (2.18\%) reported in patients undergoing ambulatory surgery in this centre.

In a recent study of 140 patients undergoing dental extractions under general anesthesia (propofol $\mathrm{N}_{2} \mathrm{O}$ /sevoflurane) in our ambulatory care unit, we noted a similarly low incidence of PONV (2.9\%) in the post anesthesia room (PAR). However, the incidence at 48 hr post-op was $28 \%$ Could the author provide further details on the incidence of PONV at $48 \mathrm{hr}$ at the Toronto hospital to allow inter institutional comparison?

Documentation of other adverse events (e.g. respiratory, CNS, cardiovascular, excessive pain and bleeding) in the PAR can be accurate assessors of surgical day care associated morbidity. However, in the case of PONV, perhaps follow-up by telephone may provide us with more realistic information?

Linda M. Collins MB BCH BAO FFARCSI

Vancouver, B.C.

\section{REPLY:}

We thank Dr. Collins for ber interest in our article. ${ }^{\prime}$ Overall, $4.6 \%$ of the patients developed PONV before dis- charge. We interviewed $30 \%$ of the patients $24 \mathrm{br}$ postoperatively and found that $9.1 \%$ of the interviewed patients suffered from PONV in the first $24 \mathrm{hr}$. We do not have data on the incidence of PONV $48 \mathrm{br}$ postoperatively. Detailed information on PONV in the same patient population were presented elsewhere. ${ }^{2}$

Determining the PONV incidence 24 or $48 \mathrm{br}$ postoperatively may provide more realistic information on total PONV than recording in-hospital PONV occurrences. Most outpatients tend to assume normal activities in the first $24 \mathrm{br}$ after surgery, which may contribute to PONV following discharge. Total PONV incidence, including the occurrence of post-discharge PONV, more appropriately reflects the long-term impact of ambulatory surgery on patients, while in-hospital PONV incidence may be more important in determining PACU cost or PACU nursing workload. In our study, we decided to follow up after the first $24 \mathrm{br}$. We expect the highest PONV incidence to occur during the first $24 \mathrm{br}$ postoperatively as residual anesthetic effect subsides with time. With a 9.1\% PONV incidence at $24 \mathrm{br}$, it is unlikely that the 48-br PONV incidence in our institute would be close to the $28 \%$ PONV incidence in Dr. Collins' institution. The case-mix of ambulatory population in our institution, 36\% underwent relatively low-risk eye surgery, may explain the difference. Undoubtedly, characteristics of the patient populations play a key role in any interinstitutional comparison.

Frances Chung FRCPC

Gabor Mezei MD PhD

Doris Tong FRCPC

Toronto, Ontario

\section{References}

1 Chung F, Mezei G, Tong D. Adverse events in ambulatory surgery. A comparison between elderly and younger patients. Can J Anesth 1999; 46: 309-21.

2 Sinclair DR, Chung F, Mezei $G$. Can postoperative nausea and vomiting be predicted? Anesthesiology 1999; 91 : 109-18.

\section{Continuous interscalene block}

To the Editor:

We read the article "A new technique of continuous interscalene nerve block" and the accompanying editorial $^{2}$ published in the Canadian Journal of Anesthesia. We approve of the suggestions made by Coleman and $\mathrm{Chan}^{2}$ to prevent damage to the catheter by the sharp end of the Tuohy needle and the recommendations not to perform interscalene block 